\title{
Design of a Fluorescence Turn-on and Label-free Aptasensor Using the Intrinsic Quenching Power of G-Quadruplex to AMT
}

\author{
Dandan Wang, ${ }^{*}$ Fenghua Geng, ${ }^{*}$ Yongxiang WAng, ${ }^{* *}, * * * \dagger$ Yu MA, ${ }^{* *}$ Guixin LI, ${ }^{* \dagger}$ Peng Qu, ${ }^{* *}$ \\ Congying SHAO, $* * *$ and Maotian $\mathrm{XU}^{* * \dagger}$ \\ *College of Chemistry and Chemical Engineering, Xinjiang Normal University, Urumqi, 830054, China \\ **Henan Key Laboratory of Biomolecular Recognition and Sensing, Henan Joint International Research \\ Laboratory of Chemo/Biosensing and Early Diagnosis of Major Diseases, School of Chemistry and \\ Chemical Engineering, Shangqiu Normal University, Shangqiu, 476000, China \\ ***College of Chemistry and Material Science, Huaibei Normal University, Huaibei, 235000, China
}

\begin{abstract}
A novel fluorescent aptasensor based on the G-quadruplex induced fluorescent quenching of psoralen and the competitive interactions between $4^{\prime}$-aminomethyl-4,5',8-trimethylpsoralen (AMT), adenosine triphosphate (ATP) and G-rich DNA functionalized split ATP aptamer was proposed. The binding of ATP to the G-rich DNA functionalized split aptamer induced a significant enhancement in fluorescence emission intensity while undergoing excitation at $340 \mathrm{~nm}$. Under the optimal conditions, the developed aptasensor showed high selectivity and good accuracy for detecting ATP. The practicality of the proposed aptasensor has been confirmed by successfully analyzing ATP in spiked human blood serum samples with satisfactory results. As far as we know, this is the first time that the intrinsic quenching ability of G-quadruplex was applied to simply construct a fluorescence turn-on and label-free aptasensor. On account of the superiority of the simplicity of the design strategy, more work is expected in the future to develop a variety of novel sensors for other important analytes using the quenching capability of G-quadruplex through reasonable designs.
\end{abstract}

Keywords Fluorescent aptasensor, G-quadruplex, split aptamer, ATP

(Received December 5, 2019; Accepted February 6, 2020; Advance Publication Released Online by J-STAGE February 14, 2020)

\section{Introduction}

Adenosine triphosphate (ATP) is one of the most critical and indispensable biomolecules with three negatively charged phosphates in living organisms. ${ }^{1}$ As the main energy provider, ATP is the most direct energy source in organisms because it releases much energy during hydrolysis. ${ }^{2}$ ATP plays an important role in various physiological and pathological processes of cells. ${ }^{3,4}$ Furthermore, abnormal ATP levels have been associated with many disease such as cancer, ${ }^{5}$ cardiovascular disease, ${ }^{6}$ Parkinson's disease, ${ }^{7}$ Alzheimer's disease ${ }^{8}$ and so on. However, ATP and adenosine diphosphate (ADP) can be rapidly transformed; cellular ATP is at a millimolar level and in dynamic equilibrium. ${ }^{9} 10$ Therefore, accurate determinations of ATP are of great significance. In particular, the challenge of establishing novel methods with a clinical application power for detecting targets in biological fluids is attractive. To date, many methods have been proposed to detect ATP, such as capillary electrophoresis (CE), ${ }^{11}$ fluorescent probes, $^{12}$ colorimetric method, ${ }^{13}$ and so forth. Unfortunately, most of these reported techniques might have unavoidable limitations such as insufficient sensitivity, time-consuming construction methods, needing complicated instrumentation, which make them

$\dagger$ To whom correspondence should be addressed.

E-mail: wangyx2006@pku.edu.cn (Y. W.); $108072291 @$ qq.com (G. L.); xumaotian@sqnu.edu.cn (M. X.) impractical to use. Among these the fluorescence-based methods are the most popular, mainly due to their high sensitivity, fast response, and the possibility of in vivo and in vitro imaging. ${ }^{14}$ It is still worthwhile to establish a fluorescence assay that can be used for ATP detection in actual clinical samples.

Aptamer is a piece of DNA or RNA sequence obtained from libraries of nucleic acid molecules which are typically obtained using systematic evolution of ligands by exponential enrichment (SELEX). ${ }^{15,16}$ Aptamers are highly specific to a variety of target substances, and are therefore widely used in the field of biosensors. Aptamers are easier to synthesize, more stable, less costly, and possess specificity and sensitivity comparable to antigen-antibody reactions. ${ }^{17}$ These advantages make aptamers a powerful tool for the detection of various target substances. Therefore, aptamers, including DNA aptamers and RNA aptamers have been widely used as the recognition unit to design aptasensors for analyte detection. ${ }^{18}$ There are mainly two strategies to design the ATP aptasensors. The first strategy is to construct the ATP aptasensors using the intact ATP aptamer. ${ }^{19,20}$ The other strategy is to construct the ATP aptasensors using split ATP aptamer fragments, in which the aptamer was split into two fragments and they could specifically bind to one another in the presence of a target. Compared to the intact aptamer, the strategy based on the split aptamer is more flexible and attracts increasing attention. ATP aptasensors have been reported and widely used to quantitatively detect ATP in solution or even in living cells..$^{21,22}$ However, these reported 

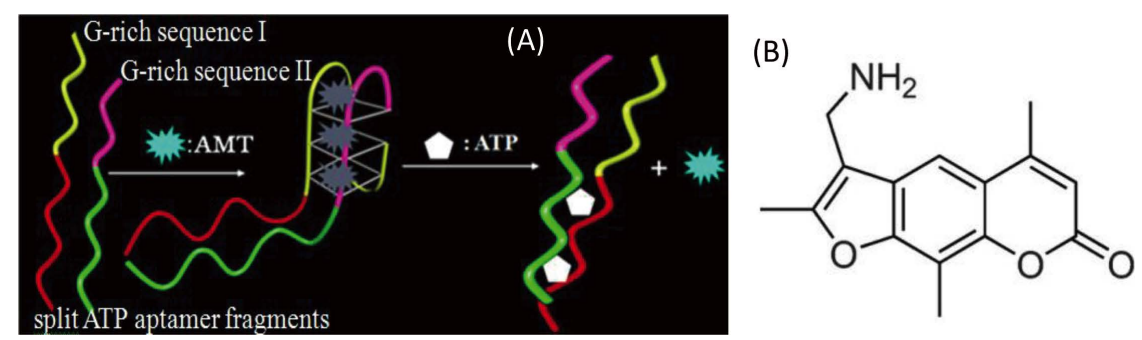

Scheme 1 (A) Schematic illustrations of the proposed fluorescence turn-on aptasensor with the G-quadruplex as a good quencher. (B) Chemical structure of AMT.

aptasensors often involve operational complexity, timeconsuming procedures, lower dynamic range, and the use of an enzyme and precious metal nanomaterial or fluorescence tagged aptamer. Therefore, the development of efficient, simple methods for the determination of ATP in a complex matrix is of considerable significance. Here, a novel strategy for designing a fluorescene turn-on and label-free aptasensor for the quantitative detection of ATP using the intrinsic quenching power of G-quadruplex was reported.

Guanine (G)-quadruplex is an atypical secondary DNA or RNA structure formed by folding G-rich DNA sequences. ${ }^{23-27}$ Although guanine has been reported as being a good quencher for fluorophore such as carboxyfluorescein (FAM), ${ }^{28,29}$ the fluorescence of many commonly used G-quadruplex ligands, such as $N$-methyl mesoporphyrin (NMM), $\alpha, \beta, \gamma, \delta$-tetrakis (1-methylpyridinium-4-yl)porphyrin $p$-toluenesulfonate (TMPyP), thioflavin T (ThT) etc., could be greatly enhanced upon binding to G-quadruplex DNA. ${ }^{30,31}$ Similar to the small organic probe, it was also found that the G-rich sequence could greatly enhance the luminescence of $\mathrm{Ag}$ nanoclusters (NCs). ${ }^{32}$ However, here has rarely been reported that G-quadruplex could quench the fluorescence of a fluorophore. In 2018, Paul and Samanta reported that the G-quadruplex formed by a $22 \mathrm{AG}$ sequence of human telomeres could quench the fluorescence of $4^{\prime}$-aminomethyl-4,5',8-trimethylpsoralen (AMT, chemical structure was shown in Scheme 1B). ${ }^{33}$ AMT is one psoralen derivative and is a planar molecule which fluoresces brightly in buffer. In addition, AMT is water-soluble, low or nontoxic and is commercially used as a drug in psoralen and ultraviolet A (PUVA) therapies. More importantly, there is a strong interaction between AMT and G-quadruplex $\left(K_{\mathrm{b}}, \sim 10^{5} \mathrm{M}^{-1}\right)$, and the fluorescence of AMT was almost completely quenched when AMT was bound to G-quadruplex DNA. The nonfluorescent G-quadruplex/AMT complexes provide possibilities for the design of G-quadruplex based turn-on fluorescence sensors. However, to our knowledge, there have been no reports on a fluorescent turn-on biosensor using the good fluorescence quenching power of G-quadruplex and AMT as the signal readout. Inspired by these pioneering studies, the aim of the present study is to demonstrate a novel fluorescence turn-on aptasensor utilizing G-quadruplex as a good quencher. As a proof-of-concept, two split ATP aptamer fragments extended with G-rich sequences were selected as a molecularrecognition unit, and AMT was the fluorescent readout. Compared with other ATP aptasensors, ${ }^{34,36}$ the novel aptasensor has several advantages other than high sensitivity, good selectivity and high accuracy. First, the strategy to design a novel label-free and turn-on fluorescence aptasensor is superiority of simplicity. The sensing system consists of only two DNA strands to recognize the target and the fluorescent indicator to report recognition events. Additionally, the two
DNA strands do not require any fluorescence tagging. The label-free fluorescent aptasensors is cost-effective and could avoid influencing the binding affinity or selectivity of the ATP aptamer. Second, the developed aptasensor takes advantage of the intrinsic quenching property of G-quadruplex and ATP induced fluorescence recovery. It is believed that the turn-on sensors are preferable to the turn-off sensors because the former could reduce the possibility of false positive signals. One of the inevitable challenges of fluorescence turn-off sensors is that the signal variation is limited to $100 \%$. Another potential weakness is that it is easy to cause negative results because it difficult to distinguish between changes in fluorescence attributed to the presence of the target and other quenchers. As a general concept, sensors based on turn-on behavior are preferable to ones displaying fluorescence quenching because turn-on sensors have comparatively better sensitivity, higher resolution as well as lower potential errors than turn-off sensors. ${ }^{37,38}$ Third, the building blocks are all commercially available and inexpensive. This has a great impact on the potential applications of the proposed sensor. Finally, encouraged by these advantages, the potential applications of the proposed aptasensor have been highlighted by analyzing ATP spiked in human blood samples with satisfactory results in view of the sensitivity, selectivity and accuracy. It can be anticipating that more fluorescent turn-on sensors constructed by the quenching and recovery of the AMT fluorescence would be developed and used to detect different kinds of targets of interest.

\section{Materials and Methods}

\section{Materials and apparatus}

4'-Aminomethyltrioxsalen hydrochloride (AMT) purchased from Sigma (China, Shanghai); Tris(hydroxymethyl)aminomethane purchased from Serva (China, Shanghai); ATP (adenosine- $5^{\prime}$-triphosphate disodium trihydrate); AMP (adenosine-5'-monophosphate disodium); CTP (cytidine-5'triphosphate), disodium salt GTP (GTP, guanosine-5'triphosphate); ADP (adenosine-5'-dlphosphate disodium salt), all purchased from Sangon Biotech Co., Ltd. (Shanghai). The solution was prepared from ultrapure water $(18.2 \mathrm{M} \Omega \cdot \mathrm{cm})$. The $\mathrm{pH}$ was measured using a lightning magnetic (Shanghai) pHS3C acidity meter. DNA, purified by HPLC, was purchased from Sangon Biotech Co., Ltd. (Shanghai), and the DNA stock solution was prepared by ultrapure water. The base sequences of DNA used in this work are given in Table S1 (Supporting Information). Blood samples from healthy volunteers were supplied by Shangqiu First People's Hospital.

\section{Fluorescence measurements}

Fluorescence spectra were measured using a Hitachi F-7000 
fluorescence spectrometer. For the fluorescence detection of ATP, different concentrations of ATP were added into a $1.0 \times 10^{-2} \mathrm{M}$ Tris- $\mathrm{HCl}(\mathrm{pH} 9.0)$ buffer solution containing $1.2 \times 10^{-5}$ M DNA $1,4.0 \times 10^{-6}$ M DNA 2, $1.2 \times 10^{-6}$ M AMT, and the solution was thoroughly stirred at room temperature. The fluorescence emission spectrum was then measured immediately. The slits for excitation and emission were both set to $5 \mathrm{~nm}$ and the scanning speed was medium $(240 \mathrm{~nm} / \mathrm{min})$. The sensing system has a fluorescence emission wavelength of 360 to $660 \mathrm{~nm}$ and an excitation wavelength of $340 \mathrm{~nm}$.

\section{Circular dichroism (CD)}

The CD spectroscopy experiment was collected from a BioLogic MOS-450 circular dichroism instrument, and the light source was always maintained in a stable high-purity nitrogen $(99.99 \%)$ gas stream. The wavelength range of the spectrum acquisition was 220 to $320 \mathrm{~nm}$. The quartz cuvette cell with an optical path of $1 \mathrm{~cm}$ was selected. The scanning rate of the instrument was set to $100 \mathrm{~nm} / \mathrm{min}$ and the response time was $2 \mathrm{~s}$. The background signal of the buffer solution has been subtracted from the CD data.

\section{Real sample assay}

Human serum was prepared according to a previous report with a minor modification. ${ }^{39}$ The serum was mixed with ethanol in a ratio of $1: 1$, mixed and placed in a refrigerator $\left(4^{\circ} \mathrm{C}\right)$ for refrigeration overnight, and taken out the next day. After centrifugation $(15000 \mathrm{r} / \mathrm{min}$ for $10 \mathrm{~min})$, the supernatant was taken in an ultrafiltration centrifuge tube (Amicon Ultra- $0.5 \mathrm{~mL}$, Millipore) with a cut-off molecular weight of $3 \mathrm{kD}$, and was centrifuged again $\left(1300 \mathrm{r} / \mathrm{min}\right.$ for $20 \mathrm{~min}$ at $\left.4^{\circ} \mathrm{C}\right)$. The filtrate was taken and frozen in a refrigerator for further use. Then, $1.2 \times 10^{-5} \mathrm{M}$ DNA $1,4.0 \times 10^{-6} \mathrm{M}$ DNA 2 and $1.2 \times 10^{-6} \mathrm{M}$ AMT and different concentrations of ATP were added to $1.0 \times$ $10^{-2} \mathrm{M}$ Tris- $\mathrm{HCl}$ (pH 9.0) buffer diluted to $1 \%$ human serum, the final concentrations of ATP were $5.0 \times 10^{-5}, 5.0 \times 10^{-4}$, $1.0 \times 10^{-3} \mathrm{M}$, and we then immediately recorded the sensing fluorescence at the emission wavelength of 360 to $660 \mathrm{~nm}$ and the excitation wavelength was $340 \mathrm{~nm}$.

\section{Results and Discussion}

\section{The hypothesized working principle}

Scheme 1 illustrates the hypothesized sensing mechanism of the suggested ATP assay. AMT, itself, can induce the 22AG into the G-quadruplex structure and the fluorescence of AMT was greatly quenched. ${ }^{40}$ The effect of the ATP aptamer on the fluorescence of the AMT was first investigated. In the presence of an intact ATP aptamer, the fluorescence of AMT could not be effectively quenched (Fig. S1, SI). As a single-stranded DNA (ssDNA), the ATP aptamer exists in an irregular curly form in the absence of ATP. Thus, upon the addition of ATP, the fluorescence recovery of AMT could not be applied to ATP detection (Fig. 1, curve a). As an alternative to using intact aptamers, aptamers could be split into two or three segments. Split aptamers have also been widely used as recognition blocks in sensor design. Next, the effect of a split ATP aptamer on the fluorescence of AMT was investigated (Fig. S2, SI). The fluorescence quenching efficiency $(Q \mathrm{e} \%)\left(\left(F_{0}-F_{\min }\right) / F_{0}=64 \%\right)$ of the split ATP aptamer to AMT was slightly higher than that of the intact ATP aptamer $\left(\left(F_{0}-F_{\min }\right) / F_{0}=54 \%\right)$. As expected, the fluorescence recovery of AMT could not be applied to ATP detection too upon the introduction of a target (Fig. 1, curve b). To effectively quench the fluorescence of AMT, each of the two

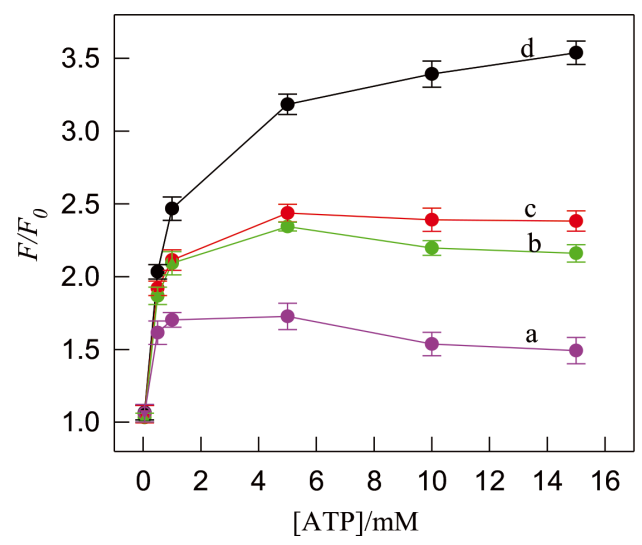

Fig. 1 Plot of the relative fluorescence intensity $\left(F / F_{0}\right)$ of the sensing system constructed with different DNA sequences versus the ATP concentration. $F$ and $F_{0}$ are the maximum fluorescence intensity of the developed aptasensor in the presence and absence of ATP, respectively.

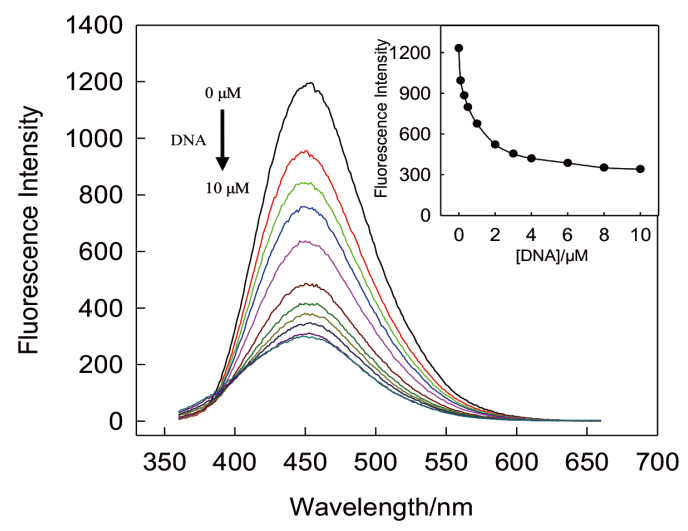

Fig. 2 Fluorescent spectra of AMT in the presence of different amounts of DNA (from top to bottom is 0, 0.1, 0.3, 0.5, 1.0, 2.0, 3.0, 4.0, 6.0, 8.0, 10.0 $\mu \mathrm{M}$ DNA 1 + DNA 2). The inset shows the evolution of the maximum fluorescent intensity $v s$. the concentration of DNA.

split ATP aptamer fragments is conjugated with a G-rich DNA sequences according to our recent studies. ${ }^{41}$ The G-rich split ATP aptamer DNA could strongly quench the fluorescence of AMT (Fig. 2). The maximum $Q \mathrm{e} \%$ was $72 \%$ in our measurements. The effective fluorescence quenching may be due to the strong interaction between AMT and G-rich DNA, so that the two G-rich DNA sequences form an intermolecular G-quadruplex. The quenched fluorescence of the AMT/G-rich DNA complex makes it possible to construct a fluorescence turn-on sensor. In the presence of ATP, the fluorescence of AMT was restored indicating that the AMT was free again in the solution (Fig. 1, curve d). It was speculated that the reason for the AMT fluorescence recovery was the binding of ATP with its split aptamer, which destabilized the AMT/G-quadruplex assembly and released free AMT into the solution. To confirm the results, two T-rich DNA sequences were labeled at one end of each of both split ATP aptamer fragments. The effect of a T-rich split ATP aptamer on the fluorescence of AMT was investigated (Fig. S3, SI). There was no noteworthy change in $Q \mathrm{e} \%$ of the T-rich split ATP aptamer (69\%) and the split ATP aptamer $(64 \%)$. Correspondingly, in the presence of ATP (Fig. 1, curve c), the fluorescence recovery of AMT was not as high as that of the G-rich split ATP aptamer. Thus, the G-rich 

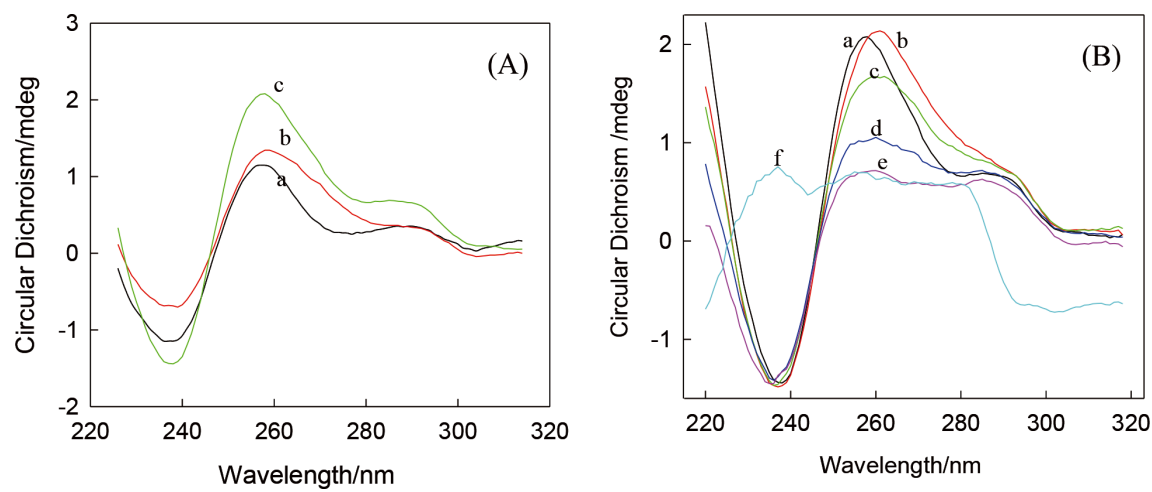

Fig. $3 \mathrm{CD}$ spectra of the sensing system in the presence of different species in $10 \mathrm{mM}$ Tris- $\mathrm{HCl}$ buffer (A) (a) $0.5 \mu \mathrm{M}$ DNA 1; (b) $0.5 \mu \mathrm{M}$ DNA 2; (c) $0.5 \mu \mathrm{M}$ DNA $1+0.5 \mu \mathrm{M}$ DNA 2. (B) (a) $0.5 \mu \mathrm{M}$ DNA $1+0.5 \mu \mathrm{M}$ DNA 2 ; (b) DNA 1/DNA $2+10 \mu \mathrm{M}$ AMT; (c) DNA 1/DNA $2 / \mathrm{AMT}+10 \mu \mathrm{M}$ ATP; (d) DNA 1/DNA 2/AMT + $30 \mu \mathrm{M}$ ATP; (e) DNA 1/DNA 2/AMT + $50 \mu \mathrm{M}$ ATP; (f) 5 mM ATP .

split ATP aptamer can strongly quench the fluorescence of AMT, probably due to the formation of the G-quadruplex/AMT complex.

\section{Investigation of the working mechanism}

The circular dichroism (CD) spectrum is widely used to characterize the secondary structure of DNA. Since the G-quadruplex has the characteristic CD signals, the CD spectrum is used here to verify the hypothesized sensing mechanism. Figure 3(A) gives the CD spectra of different DNA sequences used in this work in $1.0 \times 10^{-2} \mathrm{M}$ Tirs- $\mathrm{HCl}(\mathrm{pH} 9.0)$. The DNA 1 characteristic CD spectrum (Fig. 3(A), curve a) and the DNA 2 characteristic CD spectrum (Fig. 3(A), curve b) have a negative peak at $240 \mathrm{~nm}$, a positive peak at around $260 \mathrm{~nm}$, and the characteristic peak is a parallel G-quadruplex. This may be due to the formation of the G-quadruplex between DNA 1 or DNA 2, itself. The signal of the DNA $1+$ DNA 2 circular dichroism (Fig. 3A, curve $\mathrm{c}$ and Fig. 3B, curve a) shows that the characteristic is also a parallel G-quadruplex characteristic peak, and the signal is slightly enhanced compared with DNA 1 and DNA 2. When $1.0 \times 10^{-5} \mathrm{M}$ AMT was added to DNA 1/DNA 2 (Fig. 3(B), curve b), the CD signal of the system did not change significantly, indicating that the fluorescence quenching of AMT embedded in the G-quadruplex did not affect the configuration of the secondary structure of the system. When $1.0 \times 10^{-5}$, $3.0 \times 10^{-5}, 5.0 \times 10^{-5} \mathrm{M}$ ATP (Fig. 3(B), curves c, d, e) were gradually added to the system, the positive peak intensity of $260 \mathrm{~nm}$ in the CD spectrum gradually weakened. It may be that after adding ATP to the system, the G-rich ATP split aptamer base portion of the ATP split aptamer strongly binds, while preventing the G-rich sequence from forming the G-quadruplex, which attenuates the parallel G-quadruplex CD signal. As a control experiment, we also examined the CD spectrum in the presence of only ATP. The results showed that pure ATP had no obvious CD signal under the experimental conditions (Fig. 3(B), curve $f$ ).

\section{Optimization of the experimental conditions}

Some experiments were done to obtain the optimal experimental conditions which would influence the sensing performance. The $\mathrm{pH}$ effect on the ATP sensing was first explored over a $\mathrm{pH}$ range of $6.0-10.0$. The data show that the $\mathrm{pH}$ has little influence on the sensing performance. As displayed in Fig. S4 (SI), pH 9.0 showed the highest fluorescence enhancement $\left(F / F_{0}\right)$ for the assay of $1.66 \times 10^{-2} \mathrm{M}$ ATP

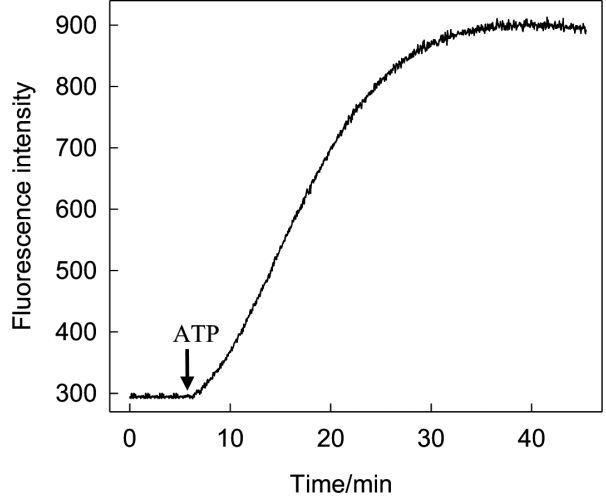

Fig. 4 Time-dependent fluorescence intensity at $445 \mathrm{~nm}$ of the developed aptasensor upon incubated with ATP.

compared to $\mathrm{pH} 6.0,7.0,8.0,9.0$ and 10.0. $F$ and $F_{0}$ are the maximum fluorescence intensity in the presence or absence of ATP, respectively. So, pH 9.0 was used in the following experiments. Monovalent cations $\left(\mathrm{K}^{+}, \mathrm{Na}^{+}\right.$, etc. $)$can affect the stability and spatial structure of the G-quadruplex. ${ }^{42}$ Subsequently, the effect of the concentration of $\mathrm{K}^{+}$on the sensing performance was studied. As can be seen from Fig. S5 (SI), $F / F_{0}$ decreased with increasing $\mathrm{K}^{+}$concentration from 0 to $0.1 \mathrm{M}$. This could be that if the G-quadruplex is too stable, the binding of ATP and the split aptamer cannot open the G-quadruplex and release the AMT. Therefore, $\mathrm{K}^{+}$is not added into the sensing system. Next, the best performance was observed when the concentration of AMT was above $1.0 \times 10^{-6} \mathrm{M}$ (Fig. S6, SI). So, $1.2 \times 10^{-6} \mathrm{M}$ AMT was selected in the sensing system. The ratio of the two split aptamer fragments also affects their binding to ATP. It can be seen from Fig. S7 (SI), that the sensing performance was best when the ratio of two segments was 3:1. This may be because the base sequence of DNA 2 is slightly longer than that of DNA 1. When the two split aptamers bind to each other in the presence of ATP, more DNA 1 strands are needed to bind to DNA 2. Finally, the effect of the concentration of the split aptamer on the sensing performance was also investigated. It can be seen from Fig. S8 (SI), that $F / F_{0}$ increased with the increasing split aptamer concentration, and reached the maximum at $1.2 \times 10^{-5} \mathrm{M}$ DNA 1 and $4.0 \times 10^{-6}$ M DNA 2 . 

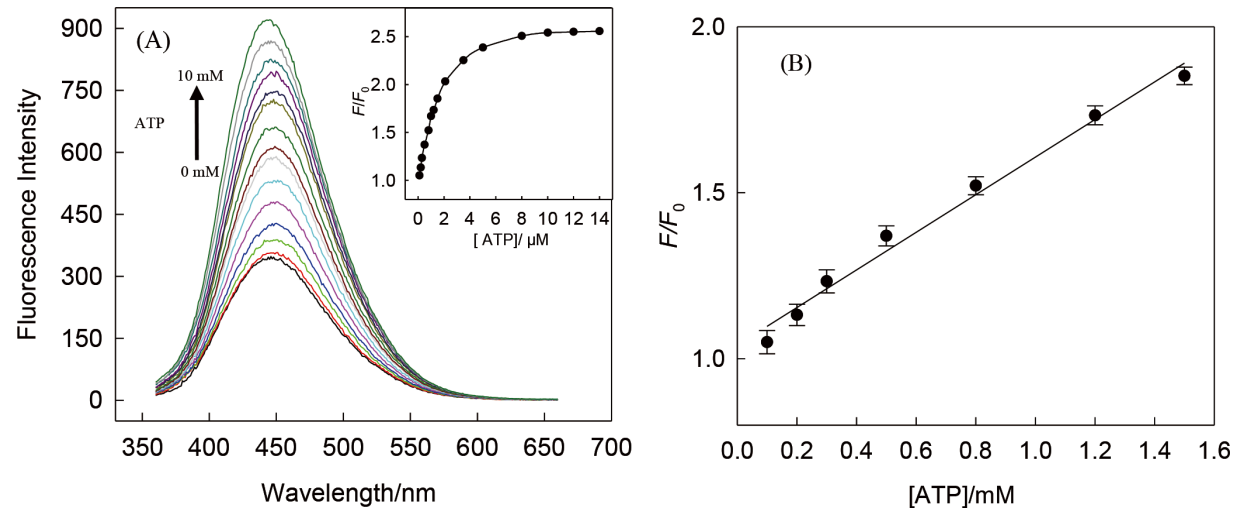

Fig. 5 (A) Fluorescence spectra of the developed aptasensor treated with varying amounts of ATP (from bottom to top: $0,0.1,0.2,0.3,0.5,0.8,1.0,1.2,1.5,2.1,2.5,3.5,5.0,8.0,10.0 \mathrm{mM}$ ). The inset shows the fluorescence intensity as a function of the ATP concentrations. (B) Linear plot of the relative fluorescence intensity $\left(F / F_{0}\right)$ as a function of the increasing concentrations of ATP. $F$ and $F_{0}$ are the maximum fluorescence intensity of the developed aptasensor in the presence and the absence of ATP, respectively.

\section{Kinetic detection of ATP in buffer solution}

The relationship between the fluorescence intensity of the sensing system and the reaction time of adding ATP is shown in Fig. 4. After adding $1.66 \times 10^{-2} \mathrm{M}$ ATP to the constructed sensing system, the fluorescence intensity of the system gradually increases with time, and regarding the fluorescence after $30 \mathrm{~min}$ of the reaction, the intensity tends to be stable. We thus chose $30 \mathrm{~min}$ as the reaction time for the system test.

\section{Detection of ATP in buffer solution}

The detection feasibility was investigated upon the addition of a varying target amount under the optimal experimental conditions. As shown in Fig. 5(A), the fluorescence intensity increased step by step as more ATP was added from $0-1.0 \times$ $10^{-2} \mathrm{M}$. As indicated in Fig. 5(A) inset, the fluorescence intensity reached the saturation point when the concentration of ATP was over $8 \times 10^{-3} \mathrm{M}$. The linear plot of $F / F_{0}$ versus the concentration of ATP (Fig. 5(B)) displayed a linear range for detecting ATP with an extraordinarily broad dynamic range from $1.0 \times 10^{-7}$ to $1.5 \times 10^{-3} \mathrm{M}$. The linear equation was $F / F_{0}=$ $0.57 c+1.04$ and the correlation coefficient $(r)$ was 0.998 . The limit of detection (LOD) was calculated to be $2.8 \times 10^{-5} \mathrm{M}$ according the $3 \sigma / k$ equation (where $\sigma$ is the standard deviation of the blank solution $(n=11)$, and $k$ represents the slope of the calibration curve).

\section{Selectivity}

To evaluate the discriminatory capability of the developed sensing system, four common ATP analogues including CTP, GTP, ADP, AMP under the same conditions were added. The results are shown in Fig. 6. Compared with ATP, the fluorescence enhancement of its analogues at the same concentration was weak. The high selectivity may be attributed to the high affinity between the aptamer and its target. Moreover, the mixture of the same concentration of ATP and its analogues was used for competitive experiments; the results showed that the analogues at the same concentration did not interfere with the ATP detection.

\section{Detection of ATP in diluted human serum}

After successfully establishing the detection of ATP in the buffer solution with the newly developed method, the potential application of the suggested method for ATP detection in

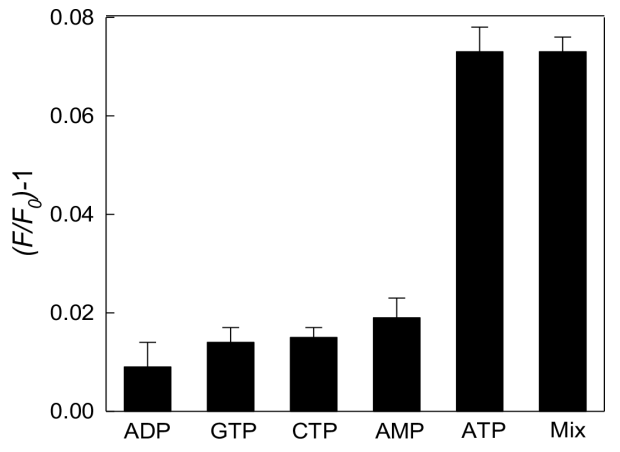

Fig. 6 Changes of the maximum fluorescence intensity of the suggested aptasensor added with the ATP or its analogs and the mixture of the substances. The standard deviation is indicated by an error bar.

Table 1 Results obtained by the newly developed method for measuring ATP in $1 \%$ human serum

\begin{tabular}{ccccc}
\hline Sample & $\begin{array}{c}\text { Add ATP/ } \\
\mathrm{mM}\end{array}$ & $\begin{array}{c}\text { Found ATP/ } \\
\mathrm{mM}\end{array}$ & $\begin{array}{c}\text { Recovery, } \\
\%\end{array}$ & $\begin{array}{c}\text { RSD, } \\
\%\end{array}$ \\
\hline 1 & 0.0 & No detect & No detect & No detect \\
2 & 1.0 & $1.076 \pm 0.052$ & 108 & 3 \\
3 & 1.2 & $1.172 \pm 0.015$ & 98 & 1 \\
\hline
\end{tabular}

practical complex human serum with the complicated matrix was validated. The accuracy of the developed method was evaluated by measuring spiked samples. As shown in Table 1 , the recoveries of the proposed method were $98-108 \%$, which are within an acceptable range. ${ }^{43,44}$ These results show that the newly developed method can be used to detect ATP in practical clinical samples.

\section{Conclusions}

In this work, taking advantage of the intrinsic quenching power of the G-quadruplex, a novel label-free, highly selective, and sensitive and fluorescence turn-on aptasensor has been successfully developed. A commercial, water-soluble and low 
toxic fluorescent indicator AMT was selected as the readout. The developed aptasensor has a LOD of $2.8 \times 10^{-5} \mathrm{M}$ and is selective toward ATP compared to its common analogues. With the developed aptasensor, the diluted human serum samples spiked with ATP were successfully analyzed. The practical application of the developed aptasensor for the measurement of ATP in real clinical complex samples was anticipated. As far as we know, this is the first time that the quenching ability of the G-quadruplex has been applied to construct a fluorescence turnon and label-free aptasensor. More studies are expected in the future to develop a variety of novel sensors for other important analytes using the quenching power of the G-quadruplex through reasonable designs. Relevant research is currently being carried out in our lab.

\section{Acknowledgements}

The authors acknowledge financial support from the National Nature Science Foundation of China (Grant Nos. 21575087), Innovation Scientists and Technicians Troop Construction Projects of Henan Province (No. 41).

\section{Supporting Information}

The base sequences of DNA used in this paper. The effect of ATP aptamer on the fluorescence of AMT. The effect of split ATP aptamer on the fluorescence of AMT. The effect of T-rich split ATP aptamer on the fluorescence of AMT. Optimization of $\mathrm{pH}$ of sensing system in $10 \mathrm{mM}$ Tris- $\mathrm{HCl}$ buffer solution. Optimization of $\mathrm{K}^{+}$concentration in sensing system. Optimization of AMT concentration in sensing system. Optimization of DNA 1/DNA 2 ratio in sensing system. Optimization of DNA concentration in sensing system. Comparison of optical sensors for ATP detection. This material is available free of charge on the Web at http://www.jsac.or.jp/ analsci/.

\section{References}

1. J. R. Knowles, Annu. Rev. Biochem., 1980, 49, 877.

2. A. Desai, S. Verma, T. J. Mitchison, and C. E. Walczak, Cell, 1999, 96, 69.

3. I. S. Kucherenko, D. Y. Didukh, O. O. Soldatkin, and A. P. Soldatkin, Anal. Chem., 2014, 86, 5455.

4. E. Llaudet, S. Hatz, M. Droniou, and N. Dale, Anal. Chem., 2005, 77, 3267.

5. D. C. Wallace, Science, 1999, 283, 1482.

6. H. Yokoshiki, M. Sunagawa, T. Seki, and N. Sperelakis, Am. J. Physiol., 1998, 274, C25.

7. Z. Zhou, Y. Du, and S. Dong, Anal. Chem., 2011, 83, 5122.

8. L. Annunziato, G. Pignataro, and G. F. DiRenzo, Pharmacol. Rev., 2004, 56, 633 .

9. R. Mo, T. Jiang, R. DiSanto, W. Tai, and Z. Gu, Nat. Commun., 2014, 5, 3364.

10. P. Peng, Y. Du, J. Zheng, H. Wang, and T. Li, Angew. Chem. Int. Ed., 2019, 58, 1648.

11. C. Fu, L. Song, and Y. Fang, Anal. Chim. Acta, 1999, 399, 259.

12. Y. X. Wang, J. S. Li, H. Wang, J. Y. Jin, J. H. Liu, K. M. Wang, W. H. Tan, and R. H. Yang, Anal. Chem., 2010, 82, 6607.

13. S. Arai, R. Kriszt, K. Harada, L. S. Looi, S. Matsuda, D.
Wongso, S. Suo, S. Ishiura, Y. H. Tseng, M. Raghunath, T. Ito, T. Tsuboi, and T. Kitaguchi, Angew. Chem. Int. Ed., 2018, 57, 10873.

14. S. Yang, C. Guo, Y. Li, J. Guo, J. Xiao, Z. Qing, J. Li, and R. Yang, ACS Sensors, 2018, 3, 2415.

15. P. L. Sazani, R. Larralde, and J. W. Szostak, J. Am. Chem. Soc., 2004, 126, 8370.

16. D. E. Huizenga and J. W.Szostak, Biochemistry, 1995, 34, 656.

17. K. Yoshimoto, Anal. Sci., 2019, 35, 1063.

18. Y. Biniuri, G.-F. Luo, M. Fadeev, V. Wulf, and I. Willner, $J$. Am. Chem. Soc., 2019, 141, 15567.

19. Y.-Y. Fan, Z.-L. Mou, M. Wang, J. Li, J. Zhang, F.-Q. Dang, and Z.-Q. Zhang, Anal. Chem., 2018, 90, 13708.

20. J. Yuan, Z. Deng, H. Liu, X. Li, J. Li, Y. He, Z. Qing, Y. Yang, and S. Zhong, ACS Sensors, 2019, 4, 1648.

21. C.-H. Zhang, H. Wang, J.-W. Liu, Y.-Y. Sheng, J. Chen, P. Zhang, and J.-H. Jiang, ACS Sensors, 2018, 3, 2526.

22. Y. W. Jun, T. Wang, S. Hwang, D. Kim, D. Ma, K. H. Kim, S. Kim, J. Jung, and K. H. Ahn, Angew. Chem. Int. Ed., 2018, 130, 10299.

23. J. Li, X. Yin, B. Li, X. Li, Y. Pan, J. Li, and Y. Guo, Anal. Chem., 2019, 91, 5354.

24. S. G. Zhang, H. X. Sun, L. X. Wang, Y. Liu, H. B. Chen, Q. Li, A. J. Guan, M. R. Liu, and Y. L. Tang, Nucleic Acids Res., 2018, 46, 7522.

25. B. L. Wang and C. Jiang, Anal. Chem., 2019, 91, 1541.

26. X. C. Chen, S. B. Chen, J. Dai, J. H. Yuan, T. M. Ou, Z. S. Huang, and J. H. Tan, Angew. Chem. Int. Ed., 2018, 57, 4702.

27. J. U. Guo and D. P. Bartel, Science, 2016, 353, aaf5371.

28. B. Datta, M. E. Bier, S. Roy, and B. A. Armitage, J. Am. Chem. Soc., 2005, 127, 4199.

29. T. Maruyama, T. Shinohara, T. Hosogi, H. Ichinose, N. Kamiya, and M. Goto, Anal. Biochem., 2006, 354, 8.

30. V. Grande, F. Doria, M. Freccero, and F. Wurthner, Angew. Chem. Int. Ed., 2017, 56, 7520.

31. J. Mohanty, N. Barooah, V. Dhamodharan, S. Harikrishna, P. I. Pradeepkumar, and A. C. Bhasikuttan, J. Am. Chem. Soc., 2013, 135, 367.

32. C. Y. Hsin, S. Jaswinder, J. H. Jason, S. M. Jennifer, and H. W. James, Nano Lett., 2010, 10, 3106.

33. S. Paul and A. Samanta, J. Phys. Chem. B, 2018, 122, 2277.

34. D. Ji, H. Wang, J. Ge, L. Zhang, J. Li, D. Bai, J. Chen, and Z. Li, Anal. Biochem., 2017, 526, 22.

35. W. Zhu, L. Dai, Z. Liu, W. Yang, C. Zhao, Y. Li, and Y. Chen, Anal. Sci., 2017, 33, 203.

36. Y. Liang, J. Su, Y. Huang, X. Li, Y. Tao, C. Lu, J. Zhu, Z. Bai, J. Meng, X. Lu, and Y. Zhao, Anal. Sci., 2016, 32, 565.

37. L. Wang, D. Su, S. N. Berry, J. Lee, and Y.-T. Chang, Chem. Commun., 2017, 53, 12465.

38. L. Mosca, S. Karimi Behzad, and P. Anzenbacher, J. Am. Chem. Soc., 2015, 137, 7967.

39. L. Zhang, Y. Hao, X. Wang, Y. Long, A. Ramos, D. Jiang, X. Ma, Q. Lin, and F. Zhou, Electroanalysis, 2015, 27, 1899.

40. S. Paul and A. Samanta, J. Phys. Chem. B, 2018, 122, 2277.

41. Y. Ma, F. Geng, Y. Wang, M. Xu, C. Shao, P. Qu, Y. Zhang, and B.Ye, Biosens. Bioelectron., 2019, 134, 36.

42. T. Li, E. Wang, and S. Dong, J. Am. Chem. Soc., 2009, 131, 15082.

43. J. Niu, X. Hu, W. Ouyang, Y. Chen, S. Liu, J. Han, and L. Liu, Anal. Chem., 2019, 91, 2360.

44. "Standard Methods for the Analysis of Water and Wastewater", 18th ed., 1992, American Public Health Association, Washington, DC. 